\title{
Análise de agrupamento de espécies lenhosas da Caatinga no Estado do Pernambuco
} \author{
José Antônio Aleixo da Silva ${ }^{3}$ \\ ${ }^{1}$ Universidade Federal de Alagoas, BR104 Norte Km 85 S/N, Mata do Rolo, CEP 57100-000, Rio Largo, AL, Brasil \\ ${ }^{2}$ Universidade Federal de Santa Maria, Av. Roraima, 1000, CEP 97105-900, Santa Maria, RS, Brasil \\ ${ }^{3}$ Universidade Federal Rural de Pernambuco, Rua Dom Manoel de Medeiros, s/n, CEP 52171-900, Recife, PE, Brasil
}

Gabriel Paes Marangon ${ }^{1}$, Roselene Marostega Felker², Anna Paula Lora Zimmermann², Rinaldo Luíz Caraciolo Ferreira ${ }^{3}$,

*Autor correspondente:

rosifelker@yahoo.com.br

Termos para indexação:

Ecologia vegetal

Twinspan

Espécies indicadoras

Index terms:

Plant ecology

Twinspan

Indicator species

Histórico do artigo:

Recebido em 08/09/2015

Aprovado em 16/09/2016

Publicado em 30/12/2016

doi: 10.4336/2016.pfb.36.88.1030

\begin{abstract}
Resumo - Este trabalho buscou analisar a formação de grupos ecológicos de espécies florestais lenhosas na caatinga, através da análise de agrupamento. Os dados são oriundos de duas áreas de estudo localizadas no município de Floresta, PE, ambas com histórico de perturbação, onde os indivíduos com circunferência a 1,30 m do solo (CAP) igual ou superior a $6,0 \mathrm{~cm}$ foram georreferenciados, identificados, e tiveram o CAP e altura mensurados. A análise de agrupamento foi realizada através do programa Twinspan no software PC-ORD. Os resultados demonstram a formação de grupos ecológicos nas duas áreas, com a presença de espécies indicadoras.
\end{abstract}

\section{Cluster analysis of Caatinga wood tree species in Pernambuco State, Brazil}

\begin{abstract}
This study aimed to analyze the formation of ecological groups of woody species in caatinga through cluster analysis. Data are from two studying areas located in Foresta, Pernambuco State, both with disturbance history. All individuals with circumference at 1.30 above ground level (CAP) greater or equal $6.0 \mathrm{~cm}$ were georeferenced, identified, and had CAP and total height measured. Cluster analysis was performed using Twinspan program in PC-ORD software. The results show the formation of ecological groups in both areas with the presence of indicator species.
\end{abstract}

\section{Introdução}

A caatinga, vegetação típica do semiárido nordestino, é um ecossistema que apresenta espécies vegetais com caracteres anatômicos, morfológicos e funcionais especializados para a sobrevivência às condições adversas de clima e solo, típicos desta fisionomia (Pereira Júnior et al., 2012), em especial a adaptação à escassez de chuvas durante a maior parte do ano. Segundo Drumond et al. (2000), a vegetação da caatinga é caracterizada por espécies de pequeno porte, geralmente dotadas de espinhos e caducifólias, representada dentre outras por cactáceas e bromeliáceas, sendo 180 espécies identificadas como endêmicas.
Apesar da importância desse ecossistema, observa-se que ainda são escassos os trabalhos sobre a vegetação do semiárido, existindo grandes lacunas no conhecimento da vegetação e de suas interações ambientais. Aliado a isto, destaca-se ainda que a caatinga se encontra em acentuado processo de degradação, provocada principalmente pelo desmatamento para ocupação de áreas por atividades agrícolas e de pecuária, assim como o uso inadequado dos recursos naturais (Drumond et al., 2000).

Estudos florísticos e fitossociológicos com observação de características ambientais são atividades que contribuem para a manutenção da biodiversidade vegetal da caatinga. Segundo Santana \& Souto (2006) existe 
uma grande preocupação atual sobre os problemas relacionados à desertificação. Esses estudos permitem monitorar as eventuais alterações na estrutura da vegetação e podem fornecer subsídios que possibilitem o aumento do conhecimento sobre o bioma (Pereira Júnior et al., 2012).

Conhecer a distribuição das espécies nas áreas de estudo pode ajudar a detectar padrões em comunidades vegetais e entender a distribuição dos indivíduos e sua relação com os fatores que influenciam a estrutura populacional (Fortin et al., 2002). Diante da dificuldade em se avaliar os processos dinâmicos de comunidades tão complexas, estudos têm sido propostos, no sentido de monitorar a dinâmica de algumas espécies nativas chaves dentro dos ecossistemas (Reis et al., 2003).

$\mathrm{O}$ conhecimento sobre o comportamento ecológico e sucessional das comunidades existentes nos fragmentos florestais, assim como o conhecimento das principais espécies, podem fornecer importantes subsídios para futuras ações de conservação e restauração. De acordo com Felfili \& Rezende (2003), esses estudos permitem analisar os diferentes métodos de reconhecimento e definição de comunidades vegetais no que se refere à origem, estrutura, classificação e relações com o meio. Isso pode ser realizado por meio de técnicas multivariadas, como a análise de agrupamentos, procedimento que busca reunir objetos em grupos homogêneos.

Nesse contexto, o presente trabalho objetivou caracterizar a estrutura horizontal de duas áreas de caatinga, bem como verificar a existência de grupos de espécies florestais e espécies indicadoras nestas áreas de estudo.

\section{Material e métodos}

Este trabalho foi realizado no município de Floresta, PE, que está inserido geologicamente na Província Borborema, pertencente à unidade geoambiental da Depressão Sertaneja. Esta tipologia representa a paisagem típica do semiárido nordestino, onde predomina o relevo suave-ondulado, cortado por vales estreitos e vertentes dissecadas (Comitê Técnico Científico da Rede de Manejo Florestal da Caatinga, 2005).

A vegetação é composta por Caatinga Hiperxerófila com trechos de Floresta Caducifólia. O clima é do tipo Tropical Semiárido, de acordo com a classificação de Köppen, apresentando-se como BSh' com chuvas de verão, entre os meses de novembro a abril, precipitação média anual de 431,8 mm e temperatura média anual de $24,7^{\circ} \mathrm{C}$. Os solos da região de estudo são classificados com Luvissolo Crômico pouco profundo, com textura superficial arenosa a média (Santos et al., 2006).

Os dados foram mensurados em duas áreas distintas, distantes cerca de $300 \mathrm{~m}$ uma da outra. A área 1 possui 10 ha e encontra-se em melhor estado de conservação. Não há registro do seu histórico de uso, mas atualmente é possível observar ação antrópica, como exploração madeireira e pastagem de caprinos. A área 2 possui 20 ha, com histórico conhecido de perturbação por desmatamento com correntão, roças de algodão, pastagem de caprinos e exploração madeireira. Estas atividades cessaram há cerca de 20 anos e atualmente essa área encontra-se abandonada.

Foram instaladas 20 e 30 parcelas de $20 \mathrm{~m} \mathrm{x}$ $20 \mathrm{~m}$, respectivamente nas áreas 1 e 2, devido à proporcionalidade da área total e pela menor densidade de espécies da área 2, resultante da sua maior degradação (Marangon, 2011). Os indivíduos com circunferência a $1,30 \mathrm{~m}$ do solo (CAP) igual ou superior a $6,0 \mathrm{~cm}$ foram georreferenciados, identificados e tiveram o CAP e altura mensurados. No caso de árvore bifurcada abaixo do ponto de medição, cada fuste foi medido como se fosse uma nova árvore, conforme protocolo de medições de parcelas permanentes (Comitê Técnico Científico da Rede de Manejo Florestal da Caatinga, 2005).

\section{Análise dos dados}

A caracterização da vegetação foi realizada através da análise de agrupamento Two-way Indicator Species Analysis (Twinspan), descrito por Jongman et al. (1995) e Felfili et al. (2007), utilizando o programa computacional PC-ORD para o Windows versão 5.0 (Mccune \& Mefford, 2006). A análise por Twinspan foi realizada considerando-se autovalores $\geq 0,30$ como relevantes (30\% de variância) (Kent \& Coker, 1992; Felfili et al., 2007). Para execução da análise foi usado o nível de corte de abundância padrão do programa PC-ORD (Felfili et al., 2007). Os agrupamentos foram caracterizados segundo a distribuição horizontal das espécies na área. Espécies com menos de cinco indivíduos por parcela foram descartadas desta análise, conforme proposto por Narvaes et al. (2008).

A estrutura horizontal da vegetação nas áreas de estudo foi descrita utilizando-se os parâmetros de densidade e frequência absolutas. 


\section{Resultados e discussão}

Foram amostrados 3.190 indivíduos, sendo 981 na área 1 e 2.209 na área 2. De acordo com estes valores, as densidades absolutas nas áreas 1 e 2 foram, respectivamente, $1.226,5$ ind. $\mathrm{ha}^{-1} \mathrm{e} 1.840,8$ ind. ha ${ }^{-1}$ (Tabela 1). Estudando o mesmo parâmetro em uma vegetação na Caatinga, Rodal et al. (1992) encontraram uma densidade absoluta de 2.208 ind. ha ${ }^{-1}$, enquanto Calixto Júnior et al. (2011) obtiveram 1350 ind. ha ${ }^{-1}$ para o mesmo parâmetro, valores próximos aos encontrados nas áreas analisadas.

O melhor estado de conservação descrito através do histórico da área 1 não elevou a densidade de indivíduos nem a diversidade florística da área. Desta forma, o menor valor da densidade em relação à área 2 não pode ser explicado somente pela ação antrópica relatada para o local. Das vinte e duas espécies analisadas neste estudo, dezoito estavam presentes em ambas as áreas, sendo que Varronia leucocephala, Thiloa glaucocarpa, Croton blanchetianus e Pityrocarpa moniliformis fizeram-se ausentes na área 1, enquanto Cynophalla flexuosa e Cnidoscolus bahianus não foram encontradas na área 2.

O número de espécies encontradas nas áreas de estudo encontra-se baixo, quando comparado a outros trabalhos realizados na caatinga (Lemos \& Rodal, 2002; Alcoforado-Filho et al., 2003). Esta variação está ligada a um conjunto de fatores referente à área, tais como topografia, exposição solar, pedologia e abundância pluviométrica (Rodal et al., 1992).

Euphorbiaceae e Fabaceae foram as famílias mais representativas nas duas áreas de amostragem. A família Euphorbiaceae parece estar associada à condição de semiaridez da região nordestina, sendo uma das famílias com maior riqueza de espécies no componente arbustivo/ arbóreo da caatinga (Araújo et al., 1995, Amorim et al., 2005; Lacerda et al., 2005).

Tabela 1. Parâmetros fitossociológicos para espécies florestais lenhosas em duas áreas de Caatinga, fazenda Itapemirim, Floresta, PE.

\begin{tabular}{|c|c|c|c|c|c|}
\hline \multirow{2}{*}{ Família } & \multirow{2}{*}{ Nome científico } & \multicolumn{2}{|c|}{ DA } & \multicolumn{2}{|c|}{ FA } \\
\hline & & Área 1 & Área 2 & Área 1 & Área 2 \\
\hline \multirow[t]{2}{*}{ Anacardiaceae } & Myracrodruon urundeuva Allemão & 26,2 & 36,7 & 45,0 & 33,3 \\
\hline & Schinopsis brasiliensis Engl. & 5,0 & 10,8 & 20,0 & 16,7 \\
\hline Aporcynaceae & Aspidosperma pyrifolium Mart. & 77,5 & 30,8 & 35,0 & 20,0 \\
\hline Boraginaceae & Varronia leucocephala (Moric.) J. S. Mill. & - & 5,0 & - & 13,3 \\
\hline Burseraceae & Commiphora leptophloeos (Mart.) J. B. Gillett & 10,0 & 3,3 & 25,0 & 10,0 \\
\hline Caparaceae & Cynophalla flexuosa (L.) J. Presl & 1,2 & - & 5,0 & - \\
\hline Combretaceae & Thiloa glaucocarpa (Mart.) Eichler & - & 56,6 & - & 36,7 \\
\hline \multirow[t]{7}{*}{ Euphorbiaceae } & Croton rhamnifolius Kunth & 332,5 & 10,8 & 70,0 & 6,7 \\
\hline & Manihot glaziovii Müll. Arg. & 117,5 & 9,2 & 85,0 & 20,0 \\
\hline & Cnidoscolus bahianus (Ule) Pax \& K. Hoffm. & 36,2 & - & 45,0 & - \\
\hline & Jatropha molicima (Pohl) Baill. & 7,5 & 175,0 & 25,0 & 53,3 \\
\hline & Jatropha mutabilis (Pohl) Baill. & 6,2 & 24,2 & 20,0 & 3,3 \\
\hline & Cnidoscolus quercifolius Pohl & 6,2 & 50,8 & 15,0 & 30,0 \\
\hline & Croton blanchetianus Baill. & - & 26,7 & - & 6,7 \\
\hline \multirow[t]{8}{*}{ Fabaceae } & Poincianella bracteosa (Tul.) L. P. Queiroz & 295,0 & 978,3 & 85,0 & 100,0 \\
\hline & Mimosa ophtalmocentra Mart. ex Benth & 136,2 & 325,0 & 55,0 & 46,7 \\
\hline & Piptadenia stipulacea (Benth.) Ducke & 93,8 & 6,7 & 70,0 & 3,3 \\
\hline & Bauhinia cheilanta (Bong.) Steud. & 42,5 & 3,3 & 50,0 & 10,0 \\
\hline & Anadenanthera colubrina (Vell.) Brenan & 16,2 & 3,3 & 35,0 & 3,3 \\
\hline & Mimosa tenuiflora Benth. & 15,0 & 28,3 & 20,0 & 6,7 \\
\hline & Senna spectabilis (DC.) H. S. Irwin \& Barneby & 1,2 & 5,8 & 5,0 & 10,0 \\
\hline & Pityrocarpa moniliformis (Benth.) Luckow \& R. W. Jobson & - & 50,0 & - & 6,7 \\
\hline Total & & $1.226,25$ & $1.840,83$ & 710,0 & 436,67 \\
\hline
\end{tabular}

$\mathrm{DA}=$ densidade absoluta e FA $=$ frequência absoluta. 


\section{Análise de agrupamento (Twinspan)}

\section{Área 1}

As espécies Myracrodruon urundeuva, Commiphora leptophloeos, Cnidoscolus bahianus, Schinopsis brasiliensis, Jatropha molissima, J. mutabilis, Cnidoscolus quercifolius, Senna spectabilis e Cynophalla flexuosa não foram contabilizadas na análise de agrupamento da área 1 pois não apresentaram o número mínimo de 5 indivíduos necessários (Narvaes et al., 2008) nas 20 parcelas analisadas. A análise de agrupamento para esta área apresentou apenas uma divisão com autovalor acima de $0,3(0,4399)$, resultando na formação de dois grupos florísticos (Figura 1). Segundo Felfili \& Venturoli (2000), autovalores superiores a 0,3 indicam que as divisões têm significado ecológico.

O primeiro grupo (G1) apresenta como espécies indicadoras Mimosa ophthalmocentra e Aspidosperma pyrifolium. Estas espécies apresentaram ocorrência nula em oito parcelas (Parcelas 13, 14, 15, 16, 17, 18, 19 e 20) das 20 amostradas. No entanto, nas demais parcelas a distribuição de ambas foi uniforme.

Marangon (2011), em trabalho realizado na mesma área de estudo, constatou que $M$. ophtalmocentra apresentou padrão agregado de ocorrência e dispersão por autocoria, registrando-se alto valor de importância na área. Barbosa et al. (2012) comenta que $M$. ophthalmocentra é uma das espécies mais adaptadas ao ambiente de caatinga, sendo endêmica desse bioma (Giulietti, et al., 2002).

Aspidosperma pyrifolium foi uma das espécies com maior representatividade, em relação ao número de indivíduos na área 1. Segundo Marangon (2011), esta se faz presente na zona do sertão baixo do Ceará, Rio Grande do Norte, Pernambuco e Paraíba, em vários tipos de solos e entre pedras e rochedos, em áreas de caatinga densa ou aberta. É considerada espécie endêmica na caatinga (Maia, 2004). É uma das poucas espécies indicadas para a recuperação de áreas em processo de desertificação, por sua importância ecológica e adaptação às mais severas condições de seca e solos rasos ou pedregosos (Prado, 2005). As espécies preferenciais do grupo foram Anadenanthera colubrina, Schinopsis brasiliensis, Poincianella bracteosa, Cnidoscolus phyllacanthus, Mimosa tenuiflora, M. ophtalmocentra, Aspidosperma pyrifolium e Bauhinia cheilanta.

O segundo grupo formado na classificação foi o G2, apresentando como espécie indicadora Cnidoscolus bahianus, espécie endêmica da caatinga, pioneira e comum na floresta decídua (mata seca) (Oliveira Filho, 2008). A espécie apresentou ocorrência nula nas primeiras 8 parcelas (P1, P2, P3, P4, P5, P6, P7 e P8). No entanto, assim como as espécies indicadoras do primeiro grupo, C. bahianus também é uma das espécies mais representativas na área de estudo.

Como espécies preferenciais do G2 têm-se também Commiphora leptophloeos, Piptadenia stipulacea, Jatropha mutabilis, Piptadenia stipulacea, Croton rhamnifolius e Manihot glaziovii. Essas espécies apresentaram ocorrência nula ou baixa nas primeiras 8 parcelas amostradas na área de estudo.

\begin{tabular}{l}
\multicolumn{1}{c|}{$\mathbf{1}^{\circ}$ divisão } \\
Grupo 1
\end{tabular}

Figura 1. Análise de agrupamento das espécies lenhosas da área 1, com a formação de dois grupos florísticos. Nota: Abreviações: Anadenan (Anadenanthera colubrina); Aspidosp (Aspidosperma pyrifolium); Cnidosco (Cnidoscolus quercifolius); Commipho (Commiphora leptophloeos); Croton b (Croton blanchetianus); Croton rh (Croton rhamnifolius); Jatropha (Jatropha molíssima); Jatroph m (Jatropha mutabilis); Manihot (Manihot glaziovii); Mimosa o (Mimosa ophtalmocentra); Mimosa t (Mimosa tenuiflora); Myracrod (Myracrodum urundeuva); Piptaden (Piptadenia stipulacea); Pityroca (Pityrocarpa moniliformis); Poincian (Poincianella bracteosa); Schinop (Schinopsis brasiliensis); Senna sp (Senna spectabilis); Thiloa gla (Thiloa glaucocarpa); Varronia (Varronia leucocephala ). 
De acordo com o histórico relatado, a área 1 apresenta-se mais conservada quando comparada com a área 2. Entretanto, nota-se a ausência de indivíduos de maior diâmetro, que pode ser explicado por estes serem considerados alvo de exploração madeireira, tanto para finalidades energéticas quanto para artesanato (Marangon, 2011).

\section{Área 2}

$\mathrm{Na}$ área 2 não foram contabilizadas para análise de agrupamento as espécies Commiphora leptophloeos, Bauhinia cheilanta, Anadenanthera colubrina, Schinopsis brasiliensis, Senna spectabilis e Varronia leucocephala. Com relação à análise de agrupamento observa-se que as duas primeiras divisões apresentaram valor ecológico, com autovalores de 0,3 e 0,3227, respectivamente, resultando na formação de 3 grupos florísticos (Figura 2).

O primeiro grupo (G1) possui como indicadoras as espécies Thiloa glaucocarpa, Cnidoscolus quercifolius, Aspidosperma pyrifolium, Schinopsis brasiliensis. Estas apresentaram, de maneira geral, ocorrência mediana na área de estudo, estando presente em cinco das dez parcelas. Com destaque a espécie T. glaucocarpa, que para este grupo apresentou o maior número de indivíduos, totalizando 68 amostrados, sendo umas das espécies mais representativas na área. T. glaucocarpa é umas das espécies mais citadas em levantamentos realizados na vegetação caducifólia espinhosa e não espinhosa do nordeste brasileiro (Araújo et al., 1995).

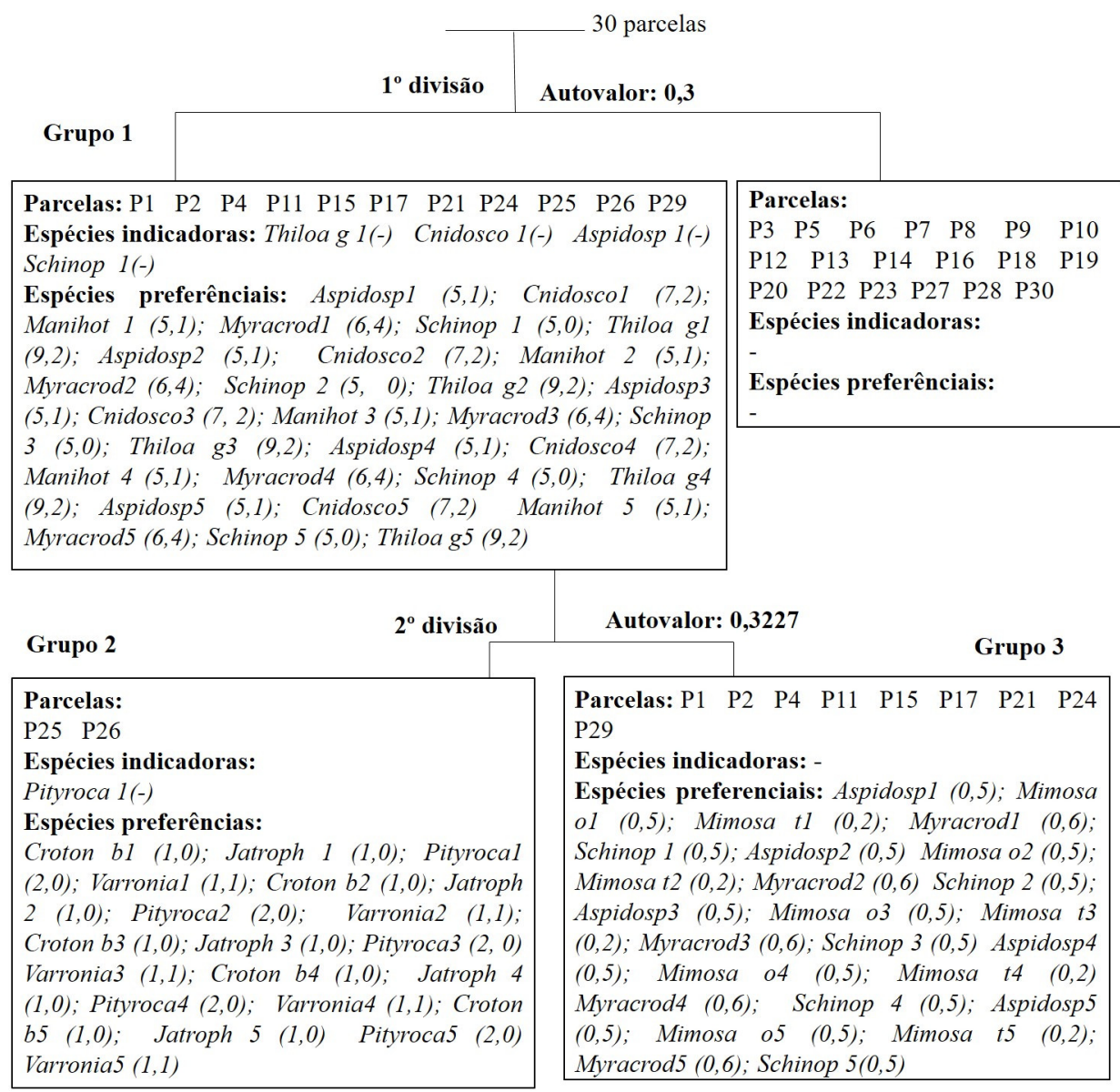

Figura 2. Análise de agrupamento das espécies lenhosas da área 2, com a formação de três grupos florísticos.

Nota: Abreviações: Anadenan (Anadenanthera colubrina); Aspidosp (Aspidosperma pyrifolium); Cnidosco (Cnidoscolus quercifolius); Commipho (Commiphora leptophloeos); Croton b (Croton blanchetianus); Croton rh (Croton rhamnifolius); Jatropha (Jatropha molíssima); Jatroph m (Jatropha mutabilis); Manihot (Manihot glaziovii); Mimosa o (Mimosa ophtalmocentra); Mimosa t (Mimosa tenuiflora); Myracrod (Myracrodum urundeuva); Piptaden (Piptadenia stipulacea); Pityroca (Pityrocarpa moniliformis); Poincian (Poincianella bracteosa); Schinop (Schinopsis brasiliensis); Senna sp (Senna spectabilis); Thiloa gla (Thiloa glaucocarpa); Varronia (Varronia leucocephala) 
Aspidosperma pyrifolium pode ser encontrada de forma espalhada na caatinga arbustiva, caatinga média, em áreas densas ou abertas (Prado, 2005). Marangon (2011) afirma que na área 2 a espécie, classificada como pioneira, obteve apenas indivíduos na primeira classe de diâmetro (indivíduos com DAP em torno de 3,4 cm), sendo a única espécie a se apresentar dessa forma. Para o autor, este seria um indício do pior estado de conservação da área 2, em relação a área 1.

Schinopsis brasiliensis é considerada dominante na Caatinga e do ponto de vista ecológico é uma das mais importantes. Apresenta baixo índice de associação, demonstrando crescer na caatinga praticamente sem afinidades com as outras espécies (Carvalho, 2009). Cnidoscolus quercifolius ocorre em todo o semiárido em regiões de caatinga (Souza et al., 2012), destacando-se na área 2 com 72,41 \% do total do valor de importância. Como preferenciais deste grupo têm-se as espécies A. pyrifolium, C. quercifolius, Manihot glaziovii, Myracrodum urundeuva, Schinopsis brasiliensis e $T$. glaucocarpa.

No grupo 2 (G2) têm-se como indicadora a espécie Pityrocarpa moniliformis. Essa espécie apresentou baixa frequência na área, sendo identificada em apenas duas, das 30 parcelas amostradas, totalizando 61 indivíduos na área analisada. É uma espécie rústica, de crescimento rápido, indicada para reflorestamentos heterogêneos (Azeredo et al., 2010). Como preferenciais desse grupo observaram-se as espécies Croton blanchetianus, Jatropha molissima, P. moniliformes e Varronia leucocephala. Estas apresentaram baixa frequência, com ocorrência em duas a três parcelas das 30 unidades amostradas.

No grupo 3 (G3), observa-se que não houve espécie indicadora, gerando apenas as espécies preferenciais (A. pyrifolium, Mimosa ophtalmocentra, M. tenuiflora, Myracrodum urundeuva e Schinopsis brasiliensis), com destaque para $M$. ophtalmocentra que totalizou 390 indivíduos amostrados.

\section{Conclusão}

A área 1 apresenta espécies indicadoras com grande número de indivíduos e distribuição agregada. A formação de diferentes grupos nas duas áreas sugere que o manejo sustentável dessas áreas deve ser diferenciado, contemplando a distribuição das espécies e as peculiaridades que cada área apresenta.
Os grupos florísticos formados concentram espécies de alta resistência e de caráter pioneiro, o que pode estar relacionado à questão antrópica e históricos relacionados às duas áreas.

\section{Referências}

Alcoforado-Filho, F. G. et al. Florística e fitossociologia de um remanescente de vegetação caducifólia espinhosa arbórea em Caruaru, Pernambuco. Acta Botânica Brasílica, v. 17, n. 2, p. 287-303, 2003. DOI: 10.1590/S0102-33062003000200011.

Amorim, I. L. et al. Flora e estrutura da vegetação arbustivo-arbórea de uma área de caatinga do Seridó, RN, Brasil. Acta Botanica Brasilica, v. 19, n. 3, p. 615-623, 2005. DOI: 10.1590/S010233062005000300023.

Araújo, E. L. et al. Composição florística e fitossociológica de três áreas de caatinga. Revista Brasileira de Biologia, v. 55, n. 4, p. $595-607,1995$.

Azeredo, G. A. et al. Superação de dormência de sementes de Piptadenia moniliformis Benth. Revista Brasileira de Sementes, v. 32, n. 2 p. 49-58, 2010. DOI: 10.1590/S0101-31222010000200006.

Barbosa, M. D. et al. Florística e fitossociologia de espécies arbóreas e arbustivas em uma área de Caatinga em Arcoverde, PE, Brasil. Revista Árvore, v. 36, n. 5, p. 851-858, 2012. DOI: 10.1590/S010067622012000500007 .

Calixto Júnior, J. T. et al. Estrutura fitossociológica de um fragmento de caatinga sensu stricto 30 anos após corte raso, Petrolina, PE, Brasil. Caatinga, v. 24, n. 2, p. 67-74, 2011.

Carvalho, P. E. R. Braúna-do-Sertão Schinopsis brasiliensis. Colombo: Embrapa Florestas, 2009. 9 p. (Embrapa Florestas. Comunicado técnico, 222).

Comitê Técnico Científico da Rede de Manejo Florestal da Caatinga. Rede de manejo florestal da Caatinga: protocolo de medições de parcelas permanentes. Recife: Associação Plantas do Nordeste, 2005. $21 \mathrm{p}$.

Drumond, M. A. et al. Estratégias para o uso sustentável da biodiversidade da caatinga. Petrolina: Embrapa Semi-Arido, 2000. 21 p. Documento para discussão em Grupo de TrabalhoGT Estratégias para o uso sustentável, apresentado no Seminário Biodiversidade da Caatinga, 2000, Petrolina.

Felfili, J. M. et al. Análise multivariada em estudos de vegetação. Brasília, DF: Universidade de Brasília, Departamento de Engenharia Florestal, 2007. 60 p.

Felfili, J. M. \& Rezende, R. P. Conceitos e métodos em fitossociologia. Brasília, DF: Universidade de Brasília, Departamento de Engenharia Florestal, 2003.

Felfili, J. M. \& Venturoli, F. Tópicos em análise de vegetação. Comunicações Técnicas Florestais, v. 2, n. 2, 2000.

Fortin, M. J. et al. Spatial analysis in ecology. In: Shaarawi, A. H. \& Piegorsch, W. W. (Ed.). Encyclopedia of environmetrics. New York: Wiley, 2002. p. 2051-2058. 
Giulietti, A. M. et al. Espécies endêmicas da caatinga. In: Sampaio, E. V. S. et al. (Ed.). Vegetação e flora da caatinga. Recife: Associação Plantas do Nordeste, 2002. p. 103-176.

Kent, M. \& Coker, P. Vegetation description and analysis. London: Wiley, 1992. $363 \mathrm{p}$.

Jongman, R. H. G. et al. Data analysis in community and landscape ecology. 2nd ed. Cambrigde: Cambrigde University Press, 1995. $299 \mathrm{p}$.

Lacerda, A. V. et al. Levantamento florístico do componente arbustivo-arbóreo da vegetação ciliar na bacia do rio Taperoá, PB, Brasil. Acta Botanica Brasilica, v. 19, n. 3, p. 647-656, 2005. DOI: 10.1590/S0102-33062005000300027.

Lemos, J. R. \& Rodal, M. J. N. Fitossociologia do componente lenhoso de um trecho da vegetação de caatinga no Parque Nacional Serra da Capivara, Piauí, Brasil. Acta Botanica Brasilica, v. 16, n. 1, p. 23-42, 2002. DOI: 10.1590/S0102-33062002000100005.

Maia, G. N. Caatinga: árvores e arbustos e suas utilidades. São Paulo: D\&Z Computação, 2004. 413 p.

Marangon, G. P. Estrutura e padrão espacial em vegetação de Caatinga. 2011.91 f. Dissertação (Mestrado em Ciências Florestais) - Universidade Federal Rural de Pernambuco, Recife.

Mccune, B. \& Mefford, M. J. PC-ORD version 5.0: multivariate analysis of ecological data. Glaneden Beach: MjM Solfware Desing, 2006.

Narvaes, I. et al. Florística e classificação da regeneração natural em floresta ombrófila mista na floresta nacional de São Francisco de Paula, RS. Revista Ciência Florestal, v. 18, p. 233-245, 2008.
Oliveira Filho, A. T et al. Espécies de ocorrência do domínio do cerrado e da caatinga. In: Oliveira Filho, A. T. \& Scolforo, J. R. (Ed.). Inventário florestal de Minas Gerais: espécies arbóreas da flora nativa. Lavras: UFLA, 2008. p. 547-575.

Pereira Júnior, L. R. et al. Composição florística e fitossociológica de um fragmento de caatinga em monteiro, PB. Holos, v. 6, 2012. DOI: 10.15628/holos.2012.1188.

Prado, D. E. As caatingas da América do Sul. In: Leal, I. R. et al. Ecologia e conservação da caatinga. Recife: Universidade Federal de Pernambuco, 2005. 822 p.

Reis, A. et. al. Restauração de áreas degradadas: a nucleação como base para os processos sucessionais. Revista Natureza \& Conservação, v. 1, n. 1, p. 28-36. 2003.

Rodal, M. J. N. et al. Manual sobre métodos de estudo florístico e fitossociológico: ecossistema caatinga. Brasília, DF: Sociedade Botânica do Brasil, 1992. 24 p.

Santana, J. A. da S. \& Souto, J. S. Diversidade e estrutura fitossociológica da caatinga na estação ecológica do Seridó, RN. Revista de Biologia e Ciência da Terra, v. 6, n. 2, p. 232-242, 2006.

Santos, H. G. dos et al. (Ed.). Sistema brasileiro de classificação de solos. 2. ed. Rio de Janeiro: Embrapa Solos, 2006. 306 p.

Souza, B. B. et al. Utilização da faveleira (Cnidoscolus phyllacanthus) como fonte de suplementação alimentar para caprinos e ovinos no semiárido brasileiro. ACSA: Agropecuária Científica no Semi-Árido, v. 8, n. 1, p. 23-27, 2012. 
